\title{
RELACIÓN ENTRE LA MINERALOGÍA DE LA FRACCIÓN ARCILLA Y LA FERTILIDAD EN ALGUNOS SUELOS CULTIVADOS CON BANANO EN LAS LLANURAS ALUVIALES DEL CARIBE DE COSTA RICA
}

\author{
Fulvio Arias ${ }^{l * *}$, Alfredo Alvarado ${ }^{* *}$, Rafael Mata ${ }^{* *}$, Edgardo Serrano $^{* * *}, J_{o r g e}$ Laguna $^{* * * * *}$ \\ Palabras clave: Mineralogía arcillas, fertilidad de suelo, Caribe, Costa Rica. \\ Keywords: Clay mineralogy, alluvial soil, Caribbean lowlands, Costa Rica.
}

Recibido: 17/11/09

\section{RESUMEN}

Se analizó la relación entre las familias mineralógicas y las características de fertilidad de suelos cultivados con banano en las llanuras del Caribe de Costa Rica. Los resultados corroboran que existen diferencias en fertilidad atribuibles al tipo y cantidad de arcilla dominante, de manera que la CIC, retención de fosfatos y las bases cambiables varían en función de la familia mineralógica. Los suelos agrupados en la familia esmectítica presentaron mayores valores de CIC y bases cambiables y menor retención de fosfatos que los suelos agrupados dentro de la familia metahaloisítica. Los suelos con presencia de vermiculita presentaron menor disponibilidad de $\mathrm{K}$ y mayor disponibilidad de $\mathrm{Mg}$.
Aceptado: $15 / 04 / 10$

\begin{abstract}
Clay mineralogy and soil fertility of banana plantation alluvial soils at Costa Rica's Caribbean lowlands. This paper analyses the relationship between soil clay mineralogy classes and soil fertility characteristics of soils cultivated with banana in the Caribbean lowlands of Costa Rica. The results corroborate that some fertility properties are closely related to the amount and type of dominant clay of the soils; among these characteristics the most relevant are CEC, phosphate retention and exchangeable cations. Soils belonging to the esmectitic clay mineralogy class showed greater values of CEC and exchangeable bases but lower phosphorus retention than soils of the metahalloysitic clay mineralogy class. Soil dominated by vermiculite showed lower amounts of available $\mathrm{K}$ and larger amounts of exchangeable $\mathrm{Mg}$.
\end{abstract}

\footnotetext{
*** CORBANA (Corporación Bananera Nacional). Guápiles, Costa Rica.

**** Escuela de Geología, Universidad de Costa Rica. San José, Costa Rica.
} 


\section{INTRODUCCIÓN}

El banano es un cultivo que crece en una amplia gama de suelos en las regiones tropicales del mundo, aunque la mejor productividad se obtiene en suelos fértiles, profundos y bien drenados, especialmente cuando se cultiva el clon Gran Enano. De tal forma que el éxito de una producción depende de la elección correcta del suelo, además del conocimiento del clima y su fertilidad química.

La región Atlántica, donde se siembra la mayor parte del banano de Costa Rica, se subdivide en las llanuras del Caribe noroeste con suelos con valores más bajos de $\mathrm{pH}, \mathrm{Ca}$, Mg, P, Mn, saturación de bases y capacidad de intercambio de cationes, pero más elevados de $\mathrm{K}$, Fe y materia orgánica y los suelos de Caribe sureste con características opuestas (Arias et al. 2010a). Además, los contenidos disponibles de los elementos menores, tienden a ser similares, considerándose que el S es deficiente en casi toda la región (Jiménez 1972, Valverde et al. 1978, Bertsch 1986, López y Solís 1991). La mayoría de estas diferencias se atribuyen al origen del material parental del cual se forman los suelos, aunque otros factores formadores de suelos juegan un papel importante (Arias et al. 2010a).

Pocos trabajos sobre suelos de la región relacionan la fertilidad de los suelos con su mineralogía. En la región noroeste de la vertiente Atlántica (Hardy 1962, Staff of the Cacao Program 1963, Hardy y Bazán 1966) indican que en la Finca La Lola (suelos aluviales de origen volcánico con mineralogía montmorillonítica en las fracciones arcilla y limo) el cacao respondió a la adición de NPK, en suelos relativamente fértiles. Los suelos alrededor de Los Diamantes (Guápiles), Penshurst, Cahuita y Limón fueron estudiados para estimar su potencial para la producción de cítricos (Krezdorn et al. 1967); los autores mencionan que la mineralogía de la fracción arcilla es principalmente amorfa, aunque a los rayos $\mathrm{X}$ también se logró identificar algo de caolinita y haloisita. Los suelos se consideraron con niveles altos de Ca y K, lo que no explicaba la marcada deficiencia de $\mathrm{Mg}$ observada en las plantaciones de cítricos, ni la alta aplicación y respuesta al $\mathrm{K}$ al cultivo del banano. El P extraído por el método de Bray y Kurtz era bajo, y lo mismo se observó para la cantidad extraída de $\mathrm{Zn}$ y Mn, deficientes en los cítricos de Los Diamantes y Guápiles.

Trabajos posteriores mencionan la existencia de problemas de disponibilidad de $\mathrm{S}$ en suelos de la vertiente Atlántica (Hardy y Bazán 1966, Pérez y Oelsligle 1975, Jaramillo y Bazán 1976, Valverde et al. 1978). Valverde et al. (1978), al estudiar los suelos de la región noroeste del Caribe, encontraron que los suelos de Roxana, Río Jiménez, Waldek y Bataán son marcadamente deficientes en S, los suelos de Siquirres, Cairo, Diamantes y Cariari tienen contenidos de S extraíble marginales y solamente en el suelo de Cariari no se encontró repuesta a la adición de $140 \mathrm{~kg} \cdot \mathrm{ha}^{-1}$ de sulfato de amonio.

Al resumir la información disponible de la época sobre génesis y formación de suelos de las llanuras de Caribe, Jiménez (1972) menciona las diferencias en fertilización entre los sectores noroeste y sureste, las cuales según Bertsch (1986) consisten en la ocurrencia de suelos ácidos y bajos en K en los cantones Siquirres y Guácimo, mientras que por el contrario, la suma de cationes es alta en los cantones Limón, Talamanca y Matina (principalmente $\mathrm{Ca}$ y $\mathrm{Mg}$ ); el contenido de $\mathrm{P}$ disponible se considera bajo en toda la provincia.

Como resultado de analizar suelos y foliares de fincas bananeras del sector noroeste (243 muestras) y sureste (403 muestras) del Caribe de Costa Rica, López y Solís (1991) concluyen que en comparación con el sector sureste, el sector noroeste tiene valores más bajos de $\mathrm{pH}(5,33$ vs $5,88), \mathrm{Ca}(5,61$ vs $25,05 \mathrm{cmol}$ (+) $100 \mathrm{~g}$ suelo), $\mathrm{Mg}$ (1,61 vs 5,98 cmol (+) 100 g suelo) y saturación de bases 25,0 vs $85,9 \mathrm{cmol}(+) 100 \mathrm{~g}$ suelo), pero más elevados de K (0,45 vs 0,36 cmol (+) $100 \mathrm{~g}$ suelo), Fe (180 vs $119 \mu \mathrm{g} . \mathrm{g}^{-1}$ suelo) y materia orgánica (5,10 vs $2,80 \%)$. Los mismos autores encontraron que los contenidos foliares de los mismos elementos siempre fueron mayores en las plantaciones del sureste y en algunos casos se consideró que los suelos de la zona noroeste contienen niveles bajos a muy bajos de $\mathrm{Ca}, \mathrm{Mg}$ y $\mathrm{Zn}$. Arias et al. 
(2010) encontró valores de $\mathrm{Ca}, \mathrm{Mg}, \mathrm{K}$, saturación de bases y MO similares a los reportados por López y Solís (1991) en estas mismas regiones. En otro estudio, Arias et al. (2003) encontraron que suelos aluviales éutricos del sureste de la vertiente Atlántica responden ligeramente a aplicaciones de $\mathrm{K}$ y no responden a las aplicaciones de Ca y $\mathrm{Mg}$; mientras que en el noroeste reportan respuesta al $\mathrm{Ca}, \mathrm{Mg}$ y $\mathrm{K}$.

En relación con la mineralogía de arcillas Arias et al. (2010b), mencionan que en las llanuras aluviales del noroeste las arcillas más abundantes son metahaloisita con trazas de materiales de bajo grado de cristalización (amorfos), gibbsita y montmorillonita; en los suelos rojos más evolucionados cerca de Colorado y en suelos meteorizados, se encuentran haloisita, gibbsita y trazas de caolinita. Las arcillas más representativas de la región sureste son las montmorillonitas, con menor ocurrencia de haloisitas, metahaloisitas y caolinitas.

En este trabajo se relaciona las diferencias en fertilidad de suelos mencionadas en la literatura, con datos propios con la mineralogía de la fracción arcilla en suelos de las llanuras aluviales de noroeste y sureste del Caribe de Costa Rica.

\section{MATERIALES Y MÉTODOS}

\section{Descripción del área y condiciones ambientales}

Esta investigación se realizó en fincas bananeras ubicadas en las cuencas de los ríos Sixaola, Estrella, Moín, Banano, Matina, Madre de Dios, Pacuare y Reventazón de las llanuras de Caribe sureste y en las cuencas Tortuguero, Chirripó Caribe y Sarapiquí de las llanuras del Caribe noroeste. De acuerdo con Holdridge (1996), las llanuras del noroeste y parte central del Caribe se ubican en las zonas de vida Bosque Muy Húmedo Tropical y las planicies del sureste en el Bosque Húmedo Tropical. La precipitación del área estudiada presenta una media de 3888 mm.año ${ }^{-1}$ con un mínimo de 3058 mm.año ${ }^{-1}$ en Sixaola y un máximo de $4789 \mathrm{~mm}^{\circ}$ año $^{-1}$ en el sector de Sarapiquí. La temperatura máxima oscila entre 31 y $30,9^{\circ} \mathrm{C}$; la media entre 24,5 y $26^{\circ} \mathrm{C}$, y la mínima entre 20,3 y $21,9^{\circ} \mathrm{C}$.

\section{Muestreo y análisis}

Para esta investigación se seleccionaron 24 fincas, distribuidas en suelos de la zona bananera de Costa Rica. Se muestreó un suelo en cada una de las cuencas de los ríos Estrella, Bananito y Moín; 2 suelos por cuenca en Sixaola, Matina, Madre de Dios y Sarapiquí; 3 suelos por cuenca en Pacuare, Reventazón y Chirripó y 4 fincas en la cuenca del Tortuguero. Arias et al. (2010a,b) describen la ubicación de los suelos estudiados. En cada finca, se abrió una calicata en la que se describieron los horizontes y sus características y se hizo un muestreo de acuerdo con los horizontes genéticos, según lineamientos de National Soil Survey Center (Versión 2 2002). La clasificación taxonómica hasta nivel de subgrupo y familia mineralógica se realizó por medio de la clave para taxonomía de suelos (Keys to Soil Taxonomy) del Soil Survey Staff (2006). El pH en $\mathrm{H}_{2} \mathrm{O}(1: 2,5)$ y la acidez intercambiable en $\mathrm{KCl} 1 \mathrm{~N}$ se determinó según metodología del Soil Survey Staff (2006). La CIC se determinó con $\mathrm{NH}_{4} \mathrm{Oac}, 1 \mathrm{~N}$, con el método de Chapman (1965); el $\mathrm{K}$, Ca y $\mathrm{Mg}$ intercambiables se extrajeron con una solución de $\mathrm{NH}_{4} \mathrm{Oac}, 1 \mathrm{~N}$, y se determinaron por medio de espectrofotometría en un equipo de con un plasma inductivamente acoplado (E-ICP); la retención de fosfatos se determinó según el método de Olsen y Sommers (1982) y el fósforo. Adicionalmente, se extrajeron el $\mathrm{Ca} \mathrm{Mg}, \mathrm{K}$ y los elementos menores con la solución Mehlich III (Mehlich 1984) y se cuantificaron por medio de espectrofotometría con un plasma inductivamente acoplado (E-ICP). La materia orgánica (MO) se determinó por medio del método de Walkey y Black, descrito por Nelson y Sommers (1982).

Para la caracterización y cuantificación de minerales en cada suelo, se tomaron muestras de cada uno de los horizontes presentes desde los 0 hasta los $60 \mathrm{~cm}$ de profundidad. De cada horizonte se tomaron muestra de aproximadamente $5 \mathrm{~kg}$, las cuales se disgregaron manualmente, se 
secaron a temperatura ambiente y se tamizaron para obtener la fracción menor a $2 \mathrm{~mm}$, antes de ser sometida a la separación de sus fracciones arena, limo y arcilla. La separación de la fracción arcilla se realizo por el método de sedimentación después de destruir los carbonatos con $\mathrm{NaOAC}$, la materia orgánica con $\mathrm{H}_{2} \mathrm{O}_{2}$ y los óxidos libres de hierro con $\mathrm{HCl} 0,001 \mathrm{M}, \mathrm{H}_{2} \mathrm{O}_{2} 30 \%$ y ditionito citrato bicarbonato (Mehra y Jackson 1960). A otras muestras se les realizaron tratamientos de disolución selectiva de aluminio, hierro y sílice en oxalato ácido de amonio $\left(\mathrm{Al}_{\mathrm{o}}, \mathrm{Fe}_{\mathrm{o}}, \mathrm{Si}_{\mathrm{o}}\right)$, y con pirofosfato de sodio $\left(\mathrm{Al}_{\mathrm{p}}, \mathrm{Fe}_{\mathrm{p}}, \mathrm{Si}_{\mathrm{p}}\right)$, de acuerdo con los procedimientos descritos por Blakemore et al. (1987); mientras que la extracción del Al y del $\mathrm{Fe}$ se hizo con ditionito citrato bicarbonato $\left(\mathrm{Al}_{\mathrm{d}}, \mathrm{Fe}_{\mathrm{d}}\right)$, según el procedimiento de Mehra y Jackson (1960).

La composición mineralógica de la fracción arcilla $(<2 \mu)$ se determinó por difractometría de rayos $\mathrm{X}$ (DRX) en muestras saturadas con $\mathrm{Mg}$ y tratadas como sigue: preparado textural simple, saturadas con Etilenglicol y tratadas a temperatura de $550^{\circ} \mathrm{C}$ (Brown y Brindley 1980). Las muestras fueron radiadas de 3 a $65^{\circ} 2 \theta$ con radiación $\mathrm{CuK} \alpha$, con monocromador de grafito en un difractómetro Philips PW1050/PW1710. Para la identificación de arcillas cristalinas se utilizó como espaciamientos basales (001) los sugeridos por Whiting y Allardice (1986), Brown y Bridley (1980), Moore y Reynols (1997), Jouseein et al. (1997) y Besoaín (1985).

Una vez identificadas las arcillas dominantes se procedió a agrupar los suelos estudiados en familias mineralógicas, lo cual permitió analizar sus características químicas en función de la mineralogía. Arias et al. (2010b) describe de forma detallada las arcillas cristalinas y familias mineralógicas presentes en la zona de estudio.

\section{RESULTADOS Y DISCUSIÓN}

La clasificación taxonómica a nivel de familia mineralógica agrupa los suelos de acuerdo a los minerales dominantes en la fracción fina
$(<0,002 \mathrm{~mm})$ y por consiguiente permite hacer predicciones acerca del comportamiento del suelo y su respuesta al manejo (Soil Survey Staff 2006).

Arias et al. (2010b), presentan la distribuión espacial de las familias mineralógicas que caracterizan las áreas de cultivo de banano en el Caribe de Costa Rica, a saber montmorrillonita,

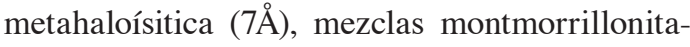
vermiculítica, montmorrillonita-metahaloísitica y montmorrillonita-vermiculítica-metahaloísitica. Desde los ríos Silencio, Vueltas y Parismina hacia el sureste la familia mineralógica dominante es la montmorrillonita, sola o mezclada con la familia vermiculítica en el sector de Pacuare, Matina y Madre de Dios o mezclada con metahaloisítica (7Å) en el sector de Sixaola. En el noroeste de los citados ríos, la familia dominante es metahaloisita (7£̊), seguida por la mezcla metahaloísitica- montmorrillonita en el sector de Sarapiquí y en mucho menor cantidad por la familia metahaloisítica-gibbsítica ubicada en las cercanías las cuencas Tortuguero y parte del Reventazón.

De acuerdo con lo indicado, para el análisis de la relación entre mineralogía y fertilidad se utilizaron como criterios de agrupación la predominancia de suelos metahaloisíticos propios de la zona Caribe noroeste y los montmorrilloníticos más relacionados con la zona Caribe sureste. Para las características de fertilidad se utilizaron datos de muestras tomadas en sitios en donde se identificó el mineral de arcilla dominante en cada una de estas zonas.

\section{Efecto de la mineralogía sobre la CIC}

La CIC de los suelos estudiados presentó diferencias entre las familias metahaloisítica y montmorrillonita (Figura 1 y Cuadros 1 y 2). De igual forma se encontró diferencias en valores de CIC de dichas familias en función del contenido de MO (Figura 2).

Conforme mayor fue la concentración de arcilla en el suelo mayor fue la CIC, tanto en suelos montmorrilloníticos como metahaloisíticos y los suelos montmorrilloníticos presentaron una CIC mayor para contenidos de arcilla similares. 


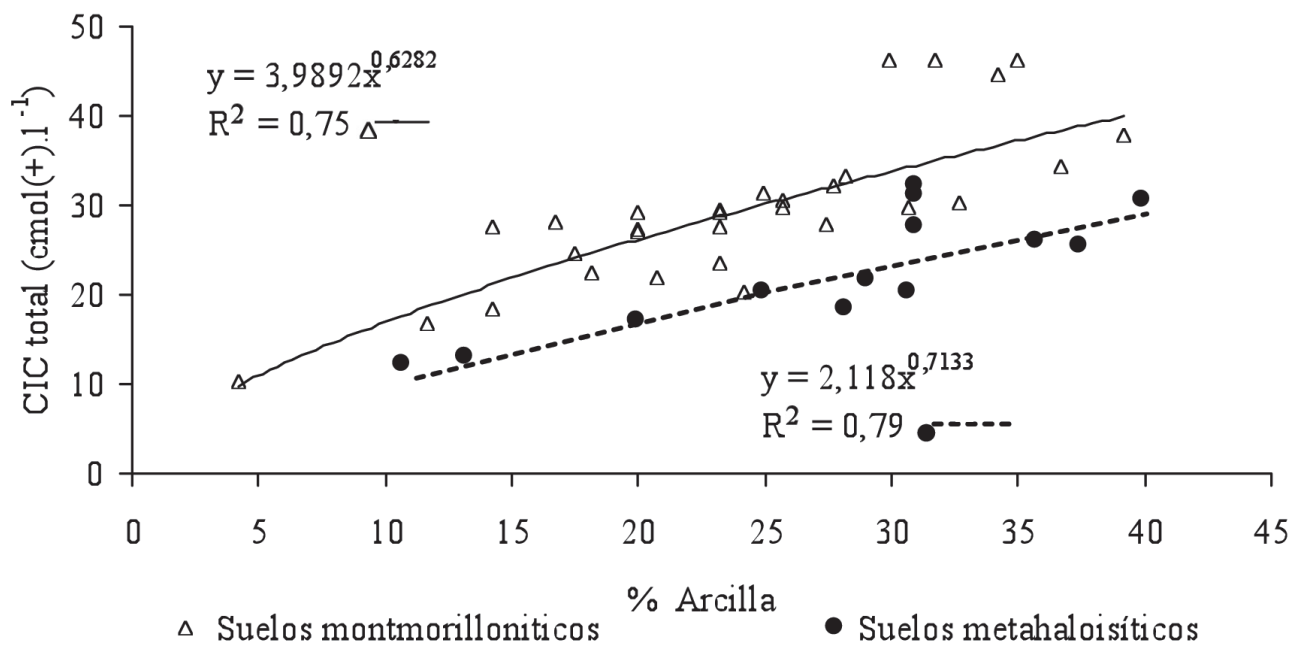

Fig. 1. Relación entre CIC y el \% de arcilla en suelos de las familias montmorillonitica y metahaloisítica.

Cuadro. 1. Características químicas en suelos de diferente familia mineralógica del sector Caribe noroeste.

\begin{tabular}{|c|c|c|c|c|c|c|c|c|c|c|c|c|c|c|c|}
\hline & \multirow[b]{2}{*}{$\begin{array}{c}\mathrm{pH} \\
\left(\mathrm{H}_{2} \mathrm{O}\right)\end{array}$} & \multirow[b]{2}{*}{$\begin{array}{c}\mathrm{pH} \\
(\mathrm{KCl})\end{array}$} & \multicolumn{4}{|c|}{$\mathrm{cmol}(+) \cdot .^{-1}$} & \multicolumn{5}{|c|}{ ppm } & \multirow[b]{2}{*}{$\begin{array}{c}\% \text { Ret. } \\
\text { Fosf. }\end{array}$} & \multirow[b]{2}{*}{$\%$ Arc. } & \multirow[b]{2}{*}{$\%$ M.O } & \multirow[b]{2}{*}{$\begin{array}{l}\operatorname{cmol}(+) \cdot 1^{-1} \\
\text { CIC suelo }\end{array}$} \\
\hline & & & $\mathrm{Ca}$ & $\mathrm{Mg}$ & $\mathrm{K}$ & $\begin{array}{c}\sum \\
\text { bases }\end{array}$ & $\mathrm{P}$ & $\mathrm{Fe}$ & $\mathrm{Cu}$ & $\mathrm{Zn}$ & $\mathrm{Mn}$ & & & & \\
\hline \multicolumn{16}{|c|}{ Metahaloisítica-Gibbsítica } \\
\hline Prom & 5,8 & 5,0 & 4,6 & 1,7 & 1,1 & 7,3 & 1 & 60 & 1 & 0 & 7 & 99 & 4,2 & 3,4 & 33,9 \\
\hline $\mathrm{N}$ & 1,0 & & & & & & & & & & & & & & \\
\hline \multicolumn{16}{|c|}{ Metahaloisítica } \\
\hline Min & 5,1 & 4,2 & 1,8 & 0,8 & 0,2 & 3,9 & 2 & 99 & 2 & 0 & 7 & 31 & 10,7 & 0,7 & 12,1 \\
\hline $\operatorname{Max}$ & 6,3 & 4,8 & 11,6 & 4,0 & 2,0 & 16,1 & 21 & 269 & 6 & 1 & 34 & 92 & 37,5 & 4,6 & 27,5 \\
\hline Prom & 6,0 & 4,5 & 8,4 & 3,0 & 1,0 & 12,2 & 8 & 178 & 3 & 1 & 16 & 60 & 26,1 & 1,8 & 20,2 \\
\hline $\mathrm{N}$ & 10,0 & & & & & & & & & & & & & & \\
\hline \multicolumn{16}{|c|}{ Metahaloisítica-Montmorillonitica } \\
\hline Min & 4,7 & 3,8 & 5,6 & 1,7 & 0,1 & 7,5 & 1 & 239 & 3 & 1 & 6 & 30 & 4,2 & 0,4 & 10,3 \\
\hline Max & 6,5 & 4,8 & 12,7 & 5,1 & 0,9 & 18,2 & 25 & 419 & 9 & 1 & 91 & 80 & 24,2 & 2,7 & 29,1 \\
\hline Prom & 5,7 & 4,4 & 9,1 & 3,1 & 0,3 & 12,5 & 9 & 318 & 4 & 1 & 34 & 55 & 16,8 & 1,6 & 19,8 \\
\hline $\mathrm{N}$ & 6,0 & & & & & & & & & & & & & & \\
\hline
\end{tabular}

El pH se determinó según Soil Survey Staff (2006); CIC con NH4Oac, 1N; Ca, Mg, K, P, Cu, Fe, Mn y Zn con Mehlich III. Retención de fosfatos con Olsen y Sommers (1982); \% de arcilla con Bouyucos; MO según método de Walkey y Black. 


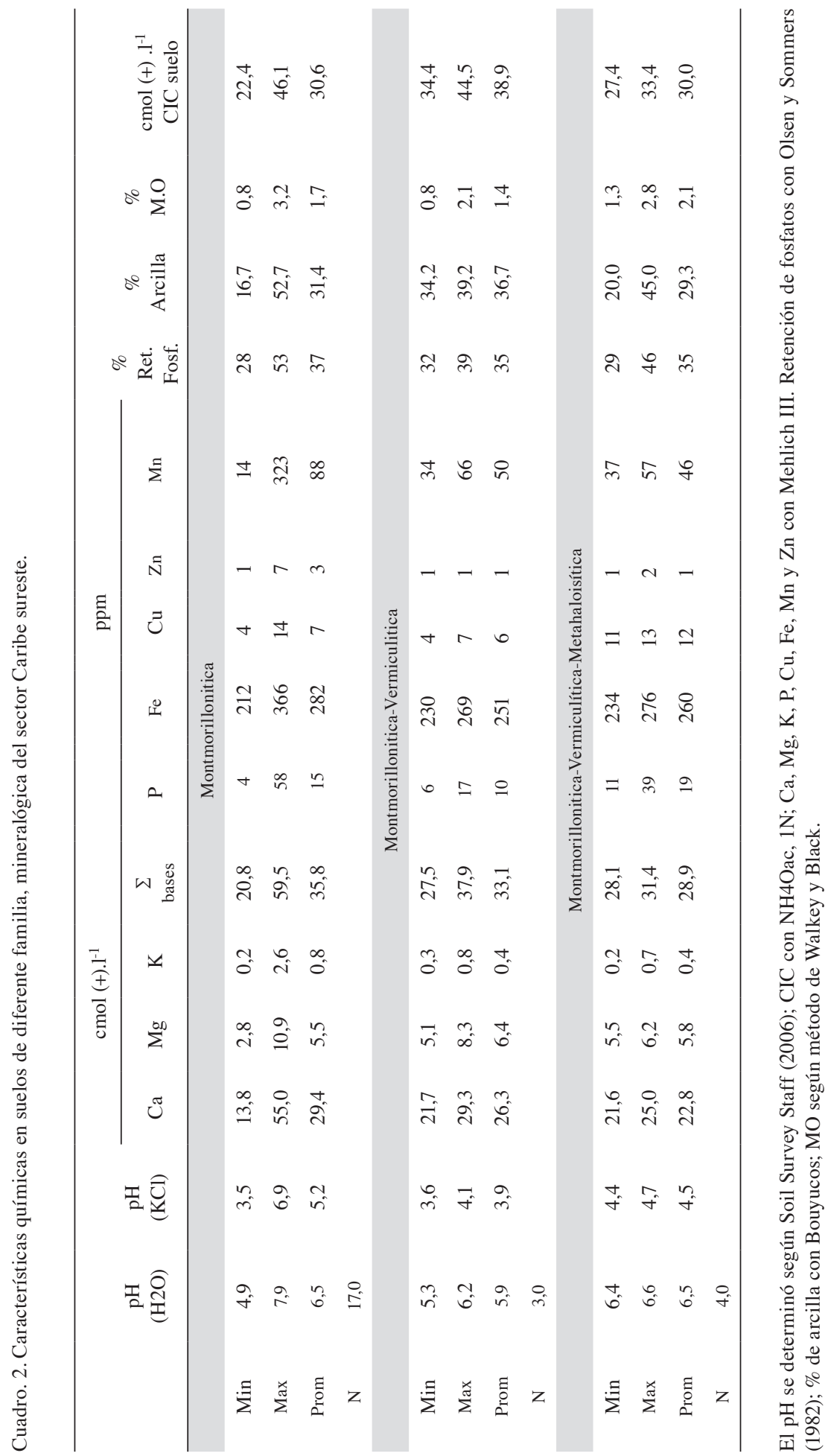




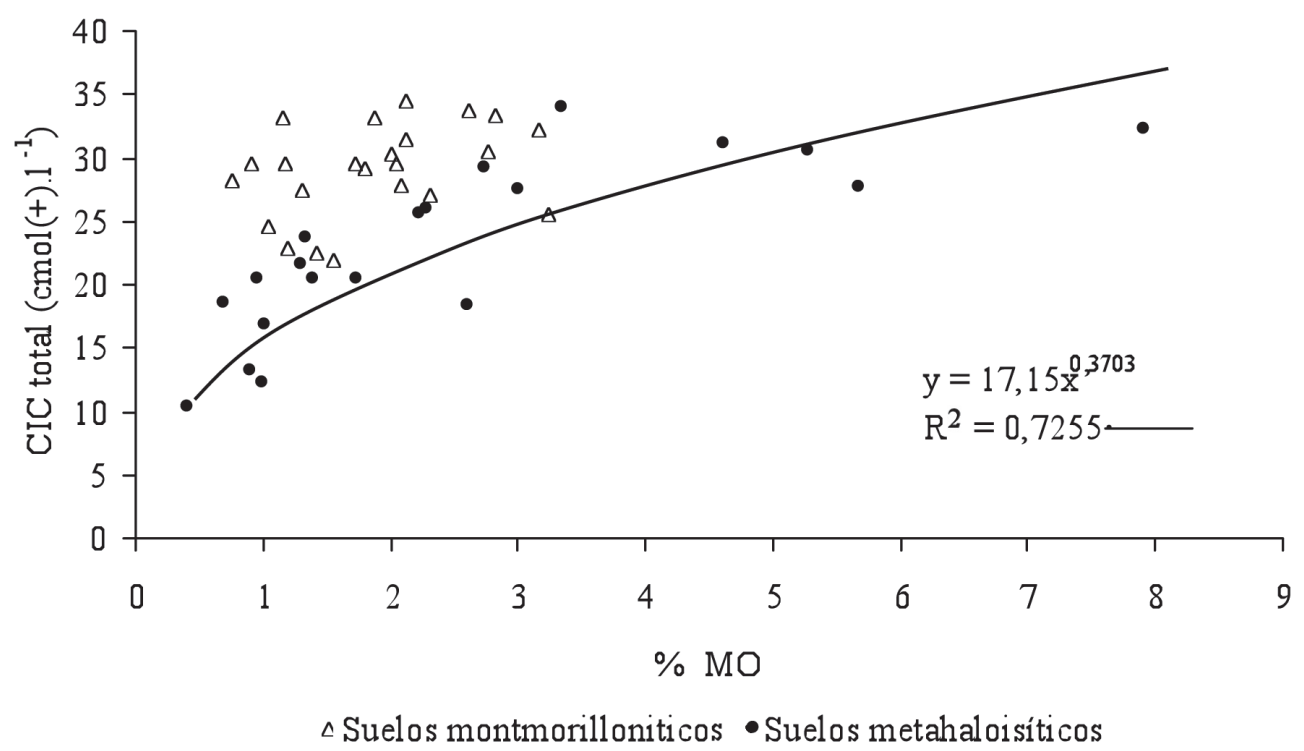

Fig. 2. Relación entre CIC y el \% de MO en suelos de las familias montmorillonitica y metahaloisítica.

En el caso de los suelos montmorrilloníticos la fracción orgánica no tiene un fuerte impacto sobre la CIC, mientras que en los suelos metahaloisíticos al aumentar el contenido de MO aumenta la CIC (Figura 2). En el caso de los suelos metahaloisíticos, la mayor cantidad de MO está relacionada con la formación de complejos organominerales de origen ándico propios de dichos suelos (Besoaín 1985), lo que no ocurre en los suelos montmorrilloníticos.

Los valores de CIC en suelos metahaloisíticos oscilan entre 12 y $29 \mathrm{cmol}(+) . l^{-1}$ y en suelos montmorrilloníticos entre 22 y 46 cmol(+). $1^{-1}$; valores similares a los reportados por Sánchez (1981) para suelos de mineralogía similar en Kenia. De acuerdo con Landon (1984) y Canchamo (1995) los suelos agrupados como metahaloisíticos tienen una CIC media y los montmorrilloníticos una CIC alta.

\section{Efecto de la mineralogía sobre las bases cambiables}

Los suelos metahaloisíticos presentan menor cantidad de bases cambiables que los suelos montmorrilloníticos (Cuadros 1 y 2), más relacionada con el tipo de arcilla que con su cantidad (Figura 3). Los suelos metahaloisíticos presentaron sumas de bases de 3,9 a 18,2 cmol(+). $\mathrm{l}^{-1}$; mientras que los suelos montmorrilloníticos presentaron valores de 20,8 a 59,5 $\mathrm{cmol}(+) . \mathrm{l}^{-1}$; que presenta las principales diferencias atribuibles a los contenidos de $\mathrm{Ca}$ y $\mathrm{Mg}$, ya que los valores de $\mathrm{K}$ son similares en ambas familias de suelos. En el caso del $\mathrm{Mg}$, los suelos con mayor presencia de vermiculita tienen mayor concentración que los suelos de las familias montmorrilloníticos y metahaloisíticas. De acuerdo con Bertsch (1986) dichos valores son medios en los suelos metahaloisíticos y altos en los montmorrilloníticos. López y Solís (1991) reportan contenidos de bases similares para ambas regiones, aunque no hacen diferencia entre familias mineralógicas; por el contrario hacen la diferencia en función de la ubicación de las fincas respecto al río Reventazón.

De acuerdo con Mejía (1984) las diferencias entre los suelos metahaloisíticos y montmorrilloníticos se deben a la estructura de dichas arcillas. Los suelos ricos en arcillas 1:1 como los metahaloisíticos tienen una superficie específica 


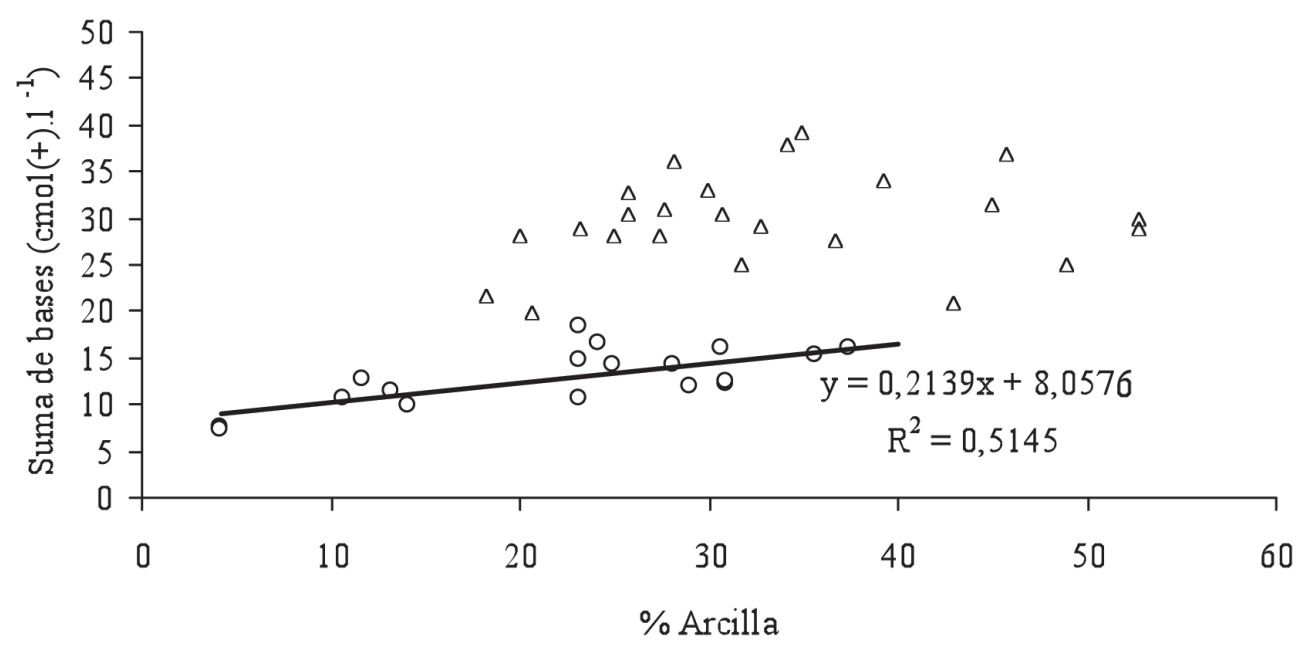

$\triangle$ Suelos montmorilloniticos $\quad$ Suelos metahaloisíticos

Fig. 3. Relación entre la sumatoria de bases y el \% de arcilla en suelos de las familias montmorillonitica y metahaloisítica.

(SE) baja $\left(5-20 \mathrm{~m}^{2} / \mathrm{g}\right)$, debido principalmente a que las unidades cristalinas están unidas por fuertes uniones $\mathrm{O}-\mathrm{OH}$; esto genera que los suelos donde predomine este mineral tengan una CIC baja, carga variable y baja saturación de bases. Por el contrario las arcillas 2:1 como la montmorillonita, tienen una SE mayor a $700 \mathrm{~m}^{2} / \mathrm{g}$ y una muy alta superficie externa e interna, debido a que las unidades cristalinas están unidas por enlaces débiles tipo O-O, los cuales permiten la expansión de las láminas y por consiguiente la entrada y salida de agua y cationes. En su estructura las montmorrillonita tienen $\mathrm{Ca}, \mathrm{Mg}$ y $\mathrm{Fe}$, lo cual hace que suelos en que estos elementos sean altos. La vermiculita, se origina por reemplazo del $\mathrm{K}$ estructural de las illitas por $\mathrm{Mg}$, lo cual implica que entre las láminas puede entrar 1 molécula de $\mathrm{H}_{2} \mathrm{O}$ en vez de 3 como en la montmorillonita; esta característica hace que la vermiculita tenga además del Ca una alta cantidad de Mg estructural y que en presencia de K en la solución de suelo, este fácilmente se fije irreversiblemente.

Aunque la disponibilidad de $\mathrm{Ca}, \mathrm{Mg}$ y $\mathrm{K}$ se explica por la mineralogía, también puede ser afectada por adiciones de fertilizantes o transferencia de elementos solubles desde la cordillera Central (Generoux y Pringle 1997, Generoux et al. 2002, Jordan 2003). Con relación a los cationes intercambiables, sus relaciones son de uso estrictamente local (Delvaux 1995), pero López (1983) sugiere para suelos bananeros rangos de 3,5 a 4 para $\mathrm{Ca} / \mathrm{Mg}, 17-25$ para $\mathrm{Ca} / \mathrm{K}$, 8-15 para $\mathrm{Mg} / \mathrm{K}, 20-38$ para $(\mathrm{Ca}+\mathrm{Mg}) / \mathrm{K}$ y Stover y Simmonds (1987) recomiendan saturaciones de Ca de $67-90 \%$ y de $2-7 \%$ para K. De acuerdo con los valores de referencia citados, los suelos con predominancia metahaloisítica tienen desbalances de $\mathrm{Ca}$, debido principalmente a altas concentraciones de $\mathrm{K}$ y en menor grado debido a altas concentraciones de $\mathrm{Mg}$ (Cuadro 3), en particular cuando existe influencia montmorrillonítica. Los suelos montmorrilloníticos, en general están más balanceados que los metahaloisíticos, lo cual se debe a que a pesar de tener concentraciones similares de $\mathrm{K}$, presentan alrededor de 2,4 veces más de Ca que los metahaloisíticos y tienen ligeros desbalances de $\mathrm{Ca}$, especialmente cuando los niveles de $\mathrm{Mg}$ son altos, como en los suelos esmectíticos-vermiculíticos. 
Cuadro 3. Relaciones de equilibrio entre $\mathrm{Ca}, \mathrm{Mg}$ y $\mathrm{K}$ en suelos del Caribe de Costa Rica, agrupados según familia mineralógica.

\begin{tabular}{|c|c|c|c|c|c|c|c|c|c|}
\hline Familia & $\mathrm{Ca}$ & $\begin{array}{c}\mathrm{cmol}^{-1} \mathrm{I}^{-1} \\
\mathrm{Mg}\end{array}$ & $\mathrm{K}$ & $\mathrm{Ca} / \mathrm{Mg}$ & $\mathrm{Ca} / \mathrm{K}$ & $\mathrm{Mg} / \mathrm{K}$ & $\mathrm{Ca}+\mathrm{Mg} / \mathrm{K}$ & $\begin{array}{c}\mathrm{Ca}^{*} 100 / \\
(\mathrm{Ca}+\mathrm{Mg}+\mathrm{K})\end{array}$ & $\begin{array}{c}\mathrm{K}^{*} 100 / \\
(\mathrm{Ca}+\mathrm{Mg}+\mathrm{K})\end{array}$ \\
\hline \multicolumn{10}{|c|}{ Sector Caribe noroeste } \\
\hline Metahaloisítica & 8,4 & 3,0 & 1,0 & 2,8 & 8,4 & 3,0 & 11,4 & 67,9 & 8,1 \\
\hline Metahaloisítica-Gibbsítica & 4,6 & 1,7 & 1,1 & 2,8 & 4,3 & 1,6 & 5,9 & 62,7 & 14,5 \\
\hline Metahaloisítica-Montmorillonitica & 9,1 & 3,1 & 0,3 & 2,9 & 32,5 & 11,2 & 43,7 & 72,8 & 2,2 \\
\hline Promedio & 7,3 & 2,6 & 0,8 & 2,8 & 15,1 & 5,2 & 20,3 & 67,8 & 8,3 \\
\hline \multicolumn{10}{|c|}{ Sector Caribe sureste } \\
\hline Montmorillonitica & 29,4 & 5,5 & 0,8 & 3,6 & 35,9 & 6,8 & 42,7 & 82,2 & 2,3 \\
\hline $\begin{array}{l}\text { Montmorillonitica- } \\
\text { Vermiculitica }\end{array}$ & 26,3 & 6,4 & 0,4 & 4,6 & 60,9 & 14,9 & 75,8 & 79,3 & 1,3 \\
\hline Mont-Verm-Metah. & 22,8 & 5,8 & 0,4 & 5,6 & 59,8 & 15,1 & 74,9 & 78,7 & 1,3 \\
\hline Promedio & 26,2 & 5,9 & 0,5 & 4,6 & 52,2 & 12,3 & 64,5 & 80,1 & 1,6 \\
\hline Valores críticos de referencia & $5^{(1)}$ & $2,3^{(1)}$ & $0,6^{(1)}$ & $3,5-4^{(2)}$ & $17-25^{(2)}$ & $8-15^{(2)}$ & $20-38^{(3)}$ & $67-90^{(3)}$ & $2-7^{(3)}$ \\
\hline
\end{tabular}

${ }^{1 /}$ Arias et al (2003), ${ }^{2 /}$ López 1983, ${ }^{3 /}$ Stover y Simmonds (1987).

\section{Efecto de la mineralogía sobre la retención de fosfatos}

La retención de fosfatos es mayor en los suelos metahaloisíticos que en los montmorrilloníticos y su intensidad está más relacionada con la cantidad de óxidos de hierro y aluminio que con la cantidad de arcilla en el suelo (Figuras 4 y 5, Cuadros 1 y 2). En los suelos metahaloisíticos la retención de fosfatos estuvo entre 31 y $99 \%$ y en los suelos montmorrilloníticos entre 28 y $57 \%$. La mayor retención en suelos metahaloisíticos se relaciona con las características ándicas de estos suelos y principalmente con la mayor ocurrencia de óxidos de Fe y Al libres extraídos con oxalato o ditionito citrato.

Respecto a la disponibilidad de P, se conoce que los suelos alofánicos y aquellos ricos en óxidos y $\mathrm{MO}$ tienen altos contenidos de $\mathrm{P}$ nativo o bien retenido y que los suelos montmorrilloníti$\cos \mathrm{y}$ vermiculiticos no poseen $\mathrm{P}$ en su estructura y tienen poca afinidad por los aniones fosfato. Los mayores contenidos de $\mathrm{P}$ en los suelos de la zona Caribe sureste no se pueden explicar debido a la mineralogía presente, lo cual permite suponer que esto ocurre por enriquecimiento con adiciones altas y sostenidas de fertilizantes fosforados. En la zona Caribe noroeste, a pesar de realizarse aplicaciones de fertilizante similares a la zona sureste, existe la posibilidad de que los fosfatos sean fijados por MO, gibbsita, alófana y otros óxidos de Fe, los cuales son más comunes en esta zona. De acuerdo con Arias (2010a) en los suelos de la zona Atlántica noroeste los \% de MO y la retención de fosfatos son mayores que en los suelos de la zona Atlántica sureste. Kamprath y Watson (1980), Sánchez (1981) y Arias et al. (2003) encontraron que la solución extractora Mehlich III, extrae menores concentraciones de P en suelos con altos valores de $\mathrm{Ca}$; lo cual explica también la mayor extracción en suelos de la zona Atlántica sureste. 


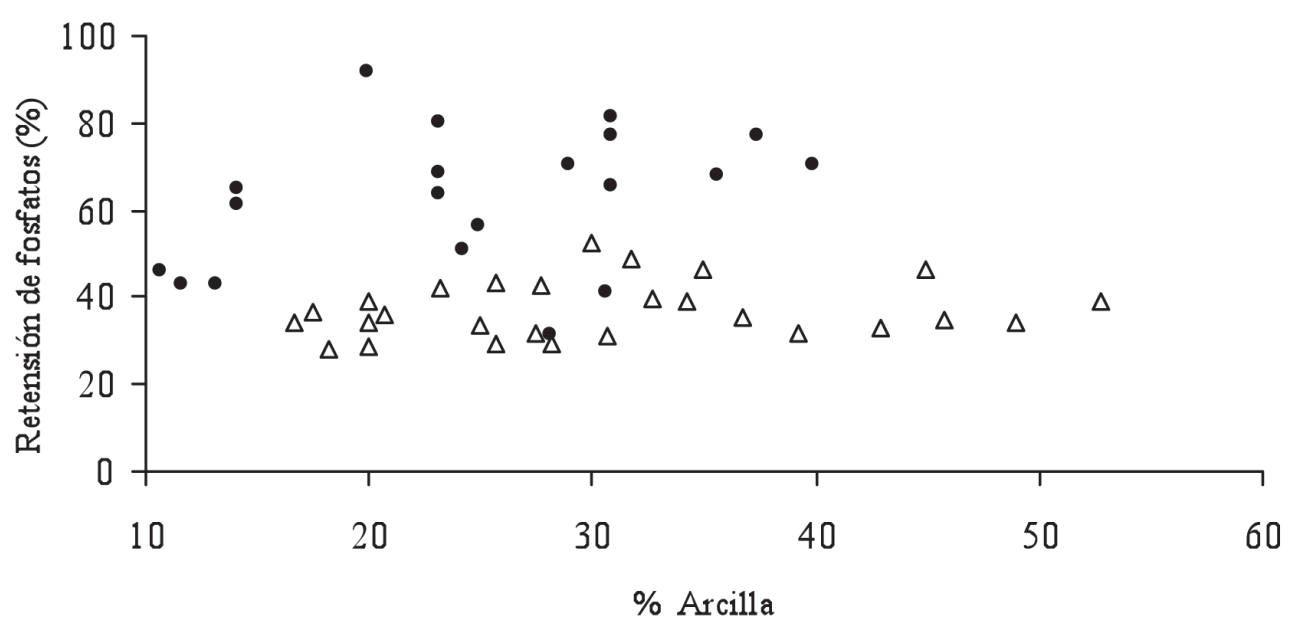

$\Delta$ Suelos montmorilloniticos $\bullet$ Suelos metahaloisíticos

Fig. 4. Relación entre \% de retención de fosfatos y el \% de arcilla en suelos de las familias montmorillonitica y metahaloisítica.

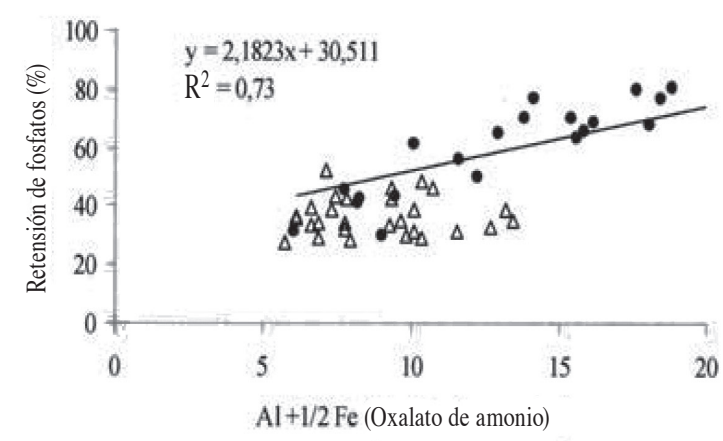

$\Delta$ Suelos montmorilloniticos $\bullet$ Suelos metahaloistíticos

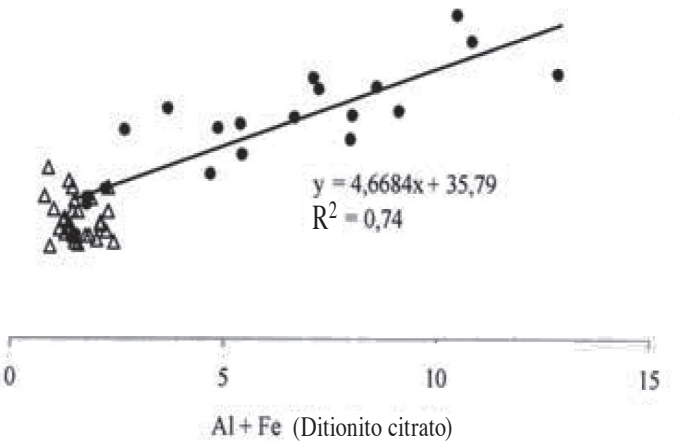

$\Delta$ Suelos montmorilloniticos $\bullet$ Suelos metahaloistíticos

Fig. 5. Relación entre \% de retención de fosfatos y la concentración de Al y Fe extraídos con Oxalato de amonio y ditionito citrato en suelos de las familias montmorillonitica y metahaloisítica. 


\section{Fertilidad del suelo y productividad}

Los resultados de esta investigación, corroboran que la fertilidad química de los suelos está definida en parte por la proporción, naturaleza (amorfa o cristalina) y la estructura específica de las arcillas en un suelo. Esto significa que de agrupar los suelos bananeros de la zona Caribe de Costa Rica según su grado de fertilidad química con relación a su mineralogía, aquellos con mayor abundancia relativa de arcillas tipo montmorillonitas y vermiculitas (arcillas 2:1) son los de mayor fertilidad y los suelos metahaloisíticos (arcillas 1:1) los de menor fertilidad (Cuadros 1, 2 y 3). No obstante, esta relación entre mineralogía y fertilidad puede ser afectada por adiciones de nutrientes vía fertilizante o bien por el aporte vía transferencia o adición de elementos solubles, producto de la meteorización de rocas en las partes altas de las laderas circundantes a través de aguas subterráneas (Generoux y Pringle 1997, Generoux et al. 2002, Jordan 2003).

Aunque se logró demostrar la relación entre fertilidad y mineralogía del suelo, no se obtuvo ninguna relación entre la productividad, y el tipo y cantidad de arcilla en los suelos. (Cuadro 4). En este caso, la escasa correlación, se explica

Cuadro. 4. Productividad (cajas.ha- ${ }^{-1}$ año ${ }^{-1}$ ) de fincas ubicadas en suelos de diferente mineralogía.

\begin{tabular}{|c|c|c|c|c|c|}
\hline \multirow{2}{*}{ Nombre de Finca } & \multirow{2}{*}{ Código } & \multirow{2}{*}{ Mineralogía } & \multirow{2}{*}{$\%$ Arcilla } & \multicolumn{2}{|c|}{ Productividad (cajas.ha $\left.{ }^{-1} \cdot a_{\tilde{n}} o^{-1}\right)^{*}$} \\
\hline & & & & Media & Máxima \\
\hline \multicolumn{6}{|c|}{ Arcillas 2:1 } \\
\hline Victoria & $\mathrm{CH}-22$ & Montmorillonitica-Metahaloisítica & 14,0 & 1993 & 2513 \\
\hline Imperio-BANDECO & PA-11 & Montmorillonitica & 19,0 & 2312 & 2442 \\
\hline Siquirres. & PA-12 & Montmorillonitica & 19,0 & 2708 & 3269 \\
\hline Catalina-SIELSA & RE-14 & Montmorillonitica & 22,0 & 2999 & 3806 \\
\hline Chiras-SIELSA & $\mathrm{CH}-21$ & Montmorillonitica-Metahaloisítica & 23,0 & 2301 & 2931 \\
\hline Fortuna-DOLE & $\mathrm{ES}-3$ & Montmorillonitica & 27,3 & 2328 & 2850 \\
\hline Penjamo & $\mathrm{CH}-20$ & Montmorillonitica-Metahaloisítica & 27,5 & 2511 & 3153 \\
\hline Nueva Esperanza-SUNISA & PA-10 & Vermiculítica & 33,0 & 1904 & 2418 \\
\hline Acumi & MA-6 & Vermiculítica & 33,0 & 3473 & 4050 \\
\hline San Pablo-CORBANA & MD-9 & Montmorillonitica & 35,0 & 2311 & 2656 \\
\hline Guaria-UNIBAN & MD-8 & Montmorillonitica -Vermiculítica & 35,5 & 2094 & 2186 \\
\hline Zent & MA-7 & Montmorillonitica -Vermiculítica & 37,0 & 2086 & 2490 \\
\hline Los Rios-DOLE & BA-4 & Montmorillonitica & 40,0 & 2950 & 3180 \\
\hline Carmen 1-BANDECO & RE-13 & Montmorillonitica & 46,0 & 2159 & 2353 \\
\hline Montebello-ACON & MO-5 & Montmorillonitica & 50,0 & 3355 & 4183 \\
\hline \multicolumn{6}{|c|}{ Arcillas 1:1 Kanditas } \\
\hline Lomas de Sierpe & TO-16 & Metahaloisítica-Gibbsítica & 6,0 & 2632 & 3035 \\
\hline Cahuita-SIELSA & TO-17 & Metahaloisítica & 14,0 & 1920 & 2111 \\
\hline Balatana & TO-18 & Metahaloisítica & 24,0 & 2495 & 2892 \\
\hline Rio Frio-DOLE & SA-24 & Metahaloisítica & 25,5 & 2169 & 2453 \\
\hline Platanera Rio Sixaola & SI-2 & Mont-Verm-Metah. & 27,0 & 2312 & 3442 \\
\hline La Rita-CORBANA & TO-19 & Metahaloisítica & 29,0 & nd & nd \\
\hline Inversiones Orlich & SA-23 & Metahaloisítica & 30,0 & 2714 & 3039 \\
\hline Banasol-ACON & RE-15 & Metahaloisítica & 31,0 & 1944 & 2194 \\
\hline Daytonia-CORBANA & SI-1 & Mont-Verm-Metah. & 42,5 & 2031 & 2410 \\
\hline
\end{tabular}

*Promedio y valor máximo de los años 1998 al 2003. 
debido a que las explotaciones bananeras consideradas para este análisis presentan cultivares, edad de plantación y manejo agronómico diferente, lo cual no permite explicar el verdadero efecto del tipo de mineralogía y su relación con la productividad como lo reportaron Stotzky y Torrence (1963) en relación con la mineralogía de arcilla, la productividad y el Mal de Panamá en suelos de América Central. Por otra parte, de acuerdo con Sánchez y Zúñiga (2005) en un reporte de estadísticas de la Corporación Bananera Nacional, durante 1994 y 2004 las fincas con mayores productividades se localizan en Limón, Matina y Siquirres, las cuales se ubican al sureste de los ríos Vueltas y Silencio y las fincas con menor productividad en los cantones de Guácimo, Pococí y Sarapiquí (donde este último cantón representa el más productivo de los 3) lo cual es explicable por ser los suelos metahaloisíticos con mayor influencia de montmorrillonita.

\section{CONCLUSIONES}

En suelos montmorrilloníticos y metahaloisíticos conforme mayor fue el contenido de arcilla mayor fue la CIC.

Para valores similares de arcillas los suelos montmorrilloníticos presentaron valores de CIC mayores que en los suelos metahaloisíticos.

En los suelos montmorrilloníticos, la fracción orgánica no tiene un fuerte impacto sobre la CIC, mientras que en los suelos metahaloisíticos al aumentar el contenido de MO aumenta la CIC.

Desde el punto de vista agronómico los suelos metahaloisíticos tienen valores medios de CIC y los montmorrilloníticos valores de CIC alta.

Los suelos metahaloisíticos presentan menor cantidad de bases cambiables que los suelos montmorrilloníticos y en ambos las diferencias son atribuibles a los contenidos de Ca y $\mathrm{Mg}$.

Los suelos con mayor contenido de vermiculita presentaron las mayores concentraciones de $\mathrm{Mg}$ y menor cantidad de $\mathrm{K}$, lo que supone fijación de este elemento.
Aunque la disponibilidad de $\mathrm{Ca}, \mathrm{Mg}$ y $\mathrm{K}$ es explicada por la mineralogía, puede ser también afectada por adiciones de fertilizantes o transferencia de elementos solubles desde la cordillera Central.

Los suelos con predominancia de arcilla metahaloisítica tienen desbalances de Ca disponible, debido principalmente a altas concentraciones de $\mathrm{K}$ disponible y en menor grado debido a altas concentraciones de $\mathrm{Mg}$ disponible y es magnificado por la influencia de la montmorrillonita.

Los suelos montmorrilloníticos, en general están más balanceados que los metahaloisíticos lo cual se debe a que a pesar de tener concentraciones similares de K, presentan alrededor de 2,4 veces más de $\mathrm{Ca}$ que los metahaloisíticos; tienen ligeros desbalances de $\mathrm{Ca}$, especialmente cuando los niveles de $\mathrm{Mg}$ son altos, como en los suelos montmorrilloníticos-vermiculíticos.

La retención de fosfatos fue mayor en los suelos metahaloisíticos que en los montmorrilloníticos y su intensidad está relacionada con las características ándicas de estos suelos y principalmente con la mayor ocurrencia de óxidos de Fe y Al libres extraídos con oxalato y ditionito citrato.

Aunque no se logró correlacionar la productividad con la mineralogía dominante, de acuerdo con estadísticas de CORBANA los suelos en donde predominan montmorrillonitas presentan en general mayor productividad que los metahaloisíticos.

\section{LITERATURA CITADA}

ARIAS F., SEGURA R., SERRANO E., BERTSCH F., LÓPEZ A., SOTO E. 2003. Niveles críticos de calcio, magnesio, potasio y fósforo en suelos dedicados al cultivo de banano (Musa AAA) en Costa Rica. CORBANA 29 (56):69-81.

ARIAS F., MATA R., ALVARADO A., SERRANO E., LAGUNA J. 2010a. Caracterización química y taxonómica de suelos cultivados con banano en las llanuras aluviales del Caribe de Costa Rica. Agronomía Costarricense 34(2): 177-195.

ARIAS F., LAGUNA J., ALVARADO A., MATA R., SERRANO E. 2010b. Mineralogía de la fracción 
arcilla de suelos cultivados con banano en las llanuras aluviales del Caribe de Costa Rica. Agronomía Costarricense 34(2): 197-222.

BERTSCH F. 1986. Manual para interpretar la fertilidad de los suelos de Costa Rica. San José, Costa Rica. Oficina de Publicaciones. UCR. 76 p.

BESOAÍN E. 1985. Mineralogía de arcillas de suelos. San José, CR, IICA. Serie de libros y materiales educativos; $\mathrm{N}^{\circ} .60 .1205 \mathrm{p}$.

BLACKMORE L.C., SEARLE P.L., DALY B.K. 1987. Methods of chemical analysis of soils. New Zealand Soil Boureau. Lower Hutt, New Zealand. Scientific Report. 80 p.

BROWN G., BRINDLEY G.W. 1980. X-ray diffraction procedures for clay mineral identification, $\mathrm{pp}$. 305-356. In: G.W. Brindley y G. Brown (ed.). Crystal structures of clay minerals and their X-ray identification. Mineral Society Monogragraph $\mathrm{N}^{\circ}$. 5. London.

CANCHAMO E. 1995. Uso y manejo de suelos. Litoflash, Santa Marta, Colombia. 305 p.

CHAPMAN H.D. 1965. Cation-exchange capacity, pp. 814901. In: C.A. Black (ed.). Methods of soil analysis: Chemical and microbiological properties. Madison, Wisconsin, American Society of Agronomy. Agronomy Series No. 9, part 2.

DELVAUX B. 1995. Soils, pp. 230-257. In: S. Gowen ed. Bananas and Plantains. London, UK, Chapman \& Hall.

GENEREUX D.P., PRINGLE C.M. 1997. Chemical and mixing model of stream flow generation at La Selva Biological Station, Costa Rica. Journal of Hydrology 199:319-330

GENEREUX D.P., WOOD S.J., PRINGLE C.M. 2002. Chemical tracing of interbasin groundwater transfer in the lowland rainforest of Costa Rica. Journal of Hydrology 258:163-178.

HARDY F. 1962. Studies in Costa Rican soils (II): (A) Senile latosol (Colorado series) and (B) La Lola farm (aluvial soil). Turrialba, Costa Rica. IICA. 7 p. (mimeo).

HARDY F., BAZÁN R. 1966. Sulfur deficiency in La Lola soils, Costa Rica. Turrialba, IICA, 6 p. (mimeo).

HOLDRIDGE L.R. 1996. Ecología basada en zonas de vida. 4ta reimpresión. San José, Costa Rica. IICA. 216 p.
JARAMILLO R., BAZÁN R. 1976. Efecto de la urea y de urea-azufre en la producción de banano "GiantCavendish" en Guápiles, Costa Rica. Turrialba 26(1):90-94.

JIMÉNEZ T. 1972. Génesis, clasificación y capacidad de uso de algunos suelos de la Región Atlántica de Costa Rica. Tesis Ingeniero Agrónomo, Universidad de Costa Rica, San José, Costa Rica. 180 p.

JORDAN M.T. 2003. Effects of water interbasin groundwater transfer on water and chemical budgets in lowland tropical watersheds-La Selva, Costa Rica. Tesis de Maestría North Carolina State University. Marine, Earth, and Atmospheric Sciences, Raleigh, North Carolina, 192 p.

JOUSSEIN E., PETIT S., CHURCHMAN J., THENG B., RIGHI D., DELVAUX B. 2005. Halloysite clay minerals-a review. Clay Minerals 40:383-426.

KAMPRATH E., WATSON M. 1980. Conventional soil and tissue tests for assessing the phosphorus status of soil, pp. 471-514. In: F.E. Khasawneth, ed. The role of phosphorus in agriculture. Madison, WS, US, ASA.

KREZDORN A.H., ROBERTSON W.K., REUSS L.A. 1967. Potential for citrus production in Costa Rica' Atlantic Zone. University of Florida/AID Mimeo Report. 31 p.

LANDON J.R. 1984. Booker Tropical Soil Manual. Booker Agriculture International Limited. London. pp. 121125 .

LÓPEZ C. 1983. Diagnóstico del estado nutricional de plantaciones bananeras. ASBANA 6(19):13-16 y 18.

LÓPEZ A., SOLÍS P. 1991. Contenidos e interacciones de los nutrimentos en tres zonas bananeras de Costa Rica. CORBANA 15(36):25-32

MEHLICH A. 1984. Mehlich 3 soil test extractant: a modification of Mehlich 2 extractant. Soil Plant Analysis 15(12):1409-1416.

MEHRA O., JACKSON M.L. 1960. Iron oxides removal from soils and clays by dithionite-citrate system buffered with sodium bicarbonate, pp. 317-327. In: Proc. 7th Clay Conf. (eds.). Clays and Clay Mineralogy. NY.

MEJÍA L. 1984. La mineralogía de los suelos y sus relaciones con la fertilidad, pp. 29-76. In: F. Silva (ed.) Fertilidad de suelos: diagnóstico y control. Bogotá, Colombia, Sociedad Colombiana de la Ciencia del Suelo. 
MOORE D.M., REYNOLDS R.C. 1997. X-Ray diffraction and the identification and analysis of clay minerals. 2 ed. Oxfort, NY, Oxfort University Press. 378 p.

NATIONAL SOIL SURVEY CENTER. 2002. Field book for describing and sampling soils (Versión 2.0). Washington, USA. Natural Resources Department of Agriculture. United States, Department of Agriculture.

NELSON D.W., SOMMERS L.E. 1982. Total carbon, organic carbon, and organic matter, pp. 539-279. In: A.L. Page, R.H. Miller, R. Keeney (eds.). Methods of soil analysis: chemical and microbiological properties. 2 ed. Madison, Wisconsin, American Society of Agronomy. Agronomy Series No. 9, part 2.

OLSEN S.R., SOMMERS L.E. 1982. Phosphorus, pp 403-430. In: A.L. Page, R.H. Miller, R. Keeney (eds.) Methods of soil analysis: chemical and microbiological properties. 2nd. ed. Madison, Wisconsin, American Society of Agronomy. Agronomy Series No. 9, part 2.

PÉREZ A., OELSLIGLE D.D. 1975. Comparación de diferentes extractantes para azufre en suelos de Costa Rica. Turrialba 25(3):232-238.

SÁNCHEZ O.R., ZÚÑIGA M.M. 2005. Costa Rica: informe anual de exportaciones de banano 2004. San José, CR. Dirección de Política Bananera y Estadísticas. Corporación Bananera Nacional S.A. 56 p.
SÁNCHEZ P. 1981. Suelos del Trópico. Características y manejo. IICA, San José, CR. 634 p.

SOIL SURVEY STAFF. 2006. Keys to soil taxonomy. 9th. ed., Washington, USA. Natural Resources Department of Agriculture. United States, Department of Agriculture. $321 \mathrm{p}$.

STAFF OF THE CACAO PROGRAM. 1963. La Lola cacao farm. Revista Cacao 8(2):1-40.

STOORVOGEL J., EPPINK G.P. 1995. Atlas de la zona Atlántica Norte de Costa Rica. Guápiles, Costa Rica. Programa Zona Atlántica (CATIE/UAV/MAG).

STOTZKY G., TORRENCE R. 1963. Soil mineralogy in relation to spread of fusarium wilt of banana in Central America. Plant and Soil 16(3):317-337.

STOVER RH., SIMMONDS NW. 1987. Bananas. 3 ed. London, UK. Longman. 468 p.

VALVERDE E., BORNEMISZA E., ALVARADO A. 1978. Disponibilidad del azufre en algunos suelos del Caribe norte de Costa Rica. Agronomía Costarricense 2(2):147-155.

WHITTIG L.D., ALLARDICE W.R. 1986. X-ray diffraction techniques, pp. 331-359. In: A Klute (ed.). Methods of soil analysis: Physical and Mineralogical Methods. 2nd ed. Madison, Wisconsin, American Society of Agronomy. Agronomy Series No. 9, part 1. 